\title{
Anatomization of various impression techniques in fixed partial prosthodontics
}

\author{
Varun Kumar,", Jyotsna Seth' ${ }^{2}$, Megha Sagar ${ }^{3}$, Vibhuti Aeran ${ }^{4}$ \\ ${ }^{1}$ Professor, ${ }^{2}$ Reader, ${ }^{3} 1{ }^{\text {st }}$ Year Post Graduate, ${ }^{4}$ BDS Final Year, Dept. of Prosthodontics, Seema Dental College \& Hospital, Rishikesh, \\ Uttarakhand, India
}

*Corresponding Author: Varun Kumar

Email: drvarun_smile@yahoo.co.in

\begin{abstract}
A good quality impression is only obtained when we have a thorough knowledge of materials, their properties and techniques for their best manipulation. In order to do so spacers are advocated for optimal thickness of impression materials in the custom tray to get the most accurate impression. There are vast number of impression techniques available in the field of Fixed Prosthodontics. Every technique has its own advantages and disadvantages, but still no impression technique is perfect with all the qualities inherent in it. The present review article discusses various impression techniques and their use in Fixed Prosthodontics.
\end{abstract}

Keywords: Spacers, Impression techniques.

\section{Introduction}

The famous aphorism aptly justifies the basics of impression making that "the perpetual preservation of what already exits and not the meticulous replacement of what is missing." Prosthodontics, as a speciality, has evolved abundantly in past few years. Materials and technological advances keep changing the face of every field every day. Over the past four decades, tremendous progress has been made in spacer designs and impression techniques for making fixed partial prostheses. ${ }^{2}$

An impression is a negative imprint of hard (teeth) and soft tissues in the mouth from which a positive reproduction can be formed. It is made by placing some soft semi-fluid material in the mouth and allowing the material to set, which is necessary to obtain a cast. This cast is obtained when an elastic material is mixed and loaded in a tray that is inserted into the patient's mouth. When the material has set, it remains elastic; the impression is then poured into the "negative" impression, and a positive likeness or definitive cast is obtained. The patient's mouth is a challenging environment to make a precise impression. With proper material selection and manipulation, accurate impression can be obtained. It must include sufficient unprepared tooth structure immediately adjacent to the margins along with all prepared surfaces in order to identify the contour of the tooth.

Many unacceptable impressions are the result of errors in various stages of the restorative procedure, such as tray selection, material of choice, improper margin location, soft tissue management etc. ${ }^{3}$ According to the literature available custom made resin trays, when properly fabricated, meet all the desired characteristics \& consequently best choice for elastomeric impression materials by limiting the volume of material, thereby reducing three sources of error: polymerization shrinkage, stress during removal and thermal contraction $^{4}$ with use of tray spacer.

Two layers of baseplate wax having thickness of $2.5 \mathrm{~mm}$ can be adapted to the diagnostic cast. To maintain the even space for impression material in the oral cavity, three stops of about $3 \times 3 \mathrm{~mm}$ should be in a tray. These are placed on nonfunctional cusps of teeth that are not to be prepared i.e. buccal cusps of maxillary teeth, lingual cusps of mandibular. If all teeth are involved, a larger soft tissue stops can be placed on the crest of the alveolar ridge or in the center of the hard palate. Stops are made by removing wax at an angle of 45 degree to the occlusal surfaces of the teeth that have a tripodal arrangement in the arch. This lends stability to the trays and the 45-degree slope helps in orientation of the tray during insertion. On the side where prepared tooth is situated, the stop should be distal to the preparation. To avoid distortion from continued polymerization of resin, the tray should be made atleast 6 to 9 hours prior to its use. The dough could shrink as much as $8 \%$ before curing is complete. Significant linear changes occur during the first 40mins. When a tray is needed more urgently it can be placed in boiling water for $5 \mathrm{~min}$. \& allowed to cool to room temperature. The impression of prepared teeth must be dimensionally accurate, stable, compatible with gypsum products and also must reproduce the surface details of prepared teeth ${ }^{5}$ all of which are prerequisite for a successful restoration.

Thus, the accuracy as well as dimensional stability of an impression may be depending on type of material used, type of tray selected and technique followed.

\section{Impression Techniques in Fixed Prosthodontics \\ I. Conventional Techniques}

A. Putty- wash Technique

a) Relieved putty impression technique.

b) Simultaneous/ Squash technique.

c) Injection Moulded putty wash technique.

B. Copper tube/Resin coping system

C. Monophase/Single viscosity technique

D. Dual viscosity technique

E. Dual arch impression technique

a) Dual-arch multiple mix technique

b) Dual-arch monophase technique

c) Laminar impression technique

d) Hydraulic pressure technique

F. Segmental impression technique

G. Wet technique

H. Hydrocolloid laminate technique 
I. Impression using polycarbonate crown

J. Functional check-bite technique

K. Sectional impressions and ' every other tooth' technique in FPD

L. Matrix impression system

II. Recent Technique

A. Digital impressions

a) chairside production

b) laboratory production

c) centralised fabrication in a production centre

\section{Conventional Techniques}

A. Putty-wash technique/ Reline Technique: The putty wash technique is commonly used for making impressions in FPD with silicone elastomers-low viscosity elastomer (lightbody or wash) against a high viscosity elastomer (putty) The putty used in a perforated metal stock tray, simulates a custom-made tray. It is recommended to overcome the problems associated with polymerization shrinkage of the condensation silicone impression materials. ${ }^{3}$ Thus impression obtained is not susceptible to distortion, impression of teeth can be captured with the wash material, while the disadvantages including more impression material required, extra chair side time and distortion.

Procedure: There are three approaches for making impression including: The first approach is to fabricate a custom tray with poly methyl methacrylate materials or lightcure materials. One layer of base plate wax is adapted over the diagnostic cast as a spacer, and wax is removed from nonfunctioning cusps to provide occlusal stops. A putty impression is made in a stock tray that results in a PVS putty custom tray. Fig. 1
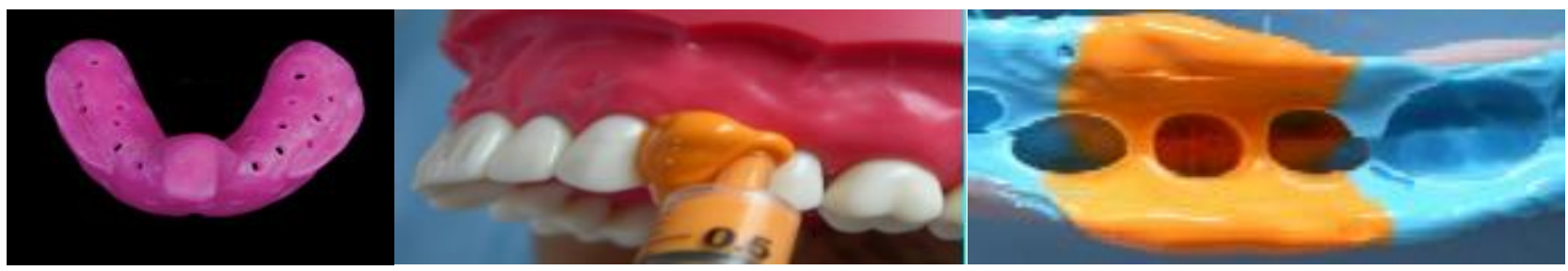

Fig. 1: Final putty impression

a) Relieved putty impression technique: In this procedure a pre-operative putty impression is made intra-orally. Gingival embrasures are prevented from material entering into them by means of plastic sheets. The characteristic of this technique is that the impression material is removed in the areas where teeth are to be prepared, using a bur or scalpel to provide 'relief' and the impression is relined/ washed using a low viscosity polyvinyl siloxane impression material. There are two potential drawbacks associated with this technique. First, the wash material in the areas of relieved impression is difficult to constrain while some of the wash material also enters the unrelieved impression resulting in erroneous occlusal pattern for the resultant cast. Secondly, there are possibilities if hydraulic distortion of the putty material as we seat the impression in the mouth. Therefore, the entire impression should be 'washed' rather than just being relieved, to eliminate inaccuracy of the impression and thus the resultant restoration.

b) Simultaneous/Squash technique: In this approach a stock tray is loaded with putty material while the syringe material is injected around the prepared tooth/teeth. This approach is objectionable since it is not possible to control the thickness of impression material and what material records the margin details of the preparation. Usually portions of the prepared margins are captured in the putty, and the putty materials are essentially deficient in their ability to record marginal details. ${ }^{6}$

c) Injection Moulded putty wash technique: In this procedure a rigid plastic tray is used to record the preoperative full arch putty impression, while the putty is prepared to make the temporary restoration. Now a buccal escape channel is created by drilling a hole through the putty from the outer surface of the tray, to allow greater flow of light body material. Once the tooth preparation is done, the tooth is cleaned \& the retraction cord is removed. The putty is then reinserted \& its position is evaluated on the tooth while the mixed light body is injected into the preparation through the holes created. While injecting the light body the tray must be held in position to prevent it from lifting off the tooth and wait for the final set. ${ }^{3}$

\section{B. Copper tube/Resin Coping System}

It is a modification of making impression with the use of impression compound. This system eliminates the use of retraction cords.

Procedure: A copper band is chosen and annealed by heating it on a fame and quenching in alcohol. The finish line is marked with the explorer while the edges are rounded off. Evaluation of the fit is done and orientation holes are then made in top one-fifth portion of the facial surface. Low fusing red stick compound is heated over the bunsen burner and the heated mass is inserted to fill approximately the top third of the copper band and then aligned onto the preparation along with compound touching just the occlusal surface. After removal of the impression, evaluate it for accuracy \& $0.2 \mathrm{~mm}$ of compound should be removed to create space for the heavy body poly vinyl silicone. A hole should be drilled through the center of the compound plug. The internal surface of the copper tube should be carefully coated with adhesive. 4-5 holes are evenly prepared just above the bottom of the copper tube for retention. The preparation is cleaned and isolated. A heavy viscosity material is mixed and injected into the band and positioned onto the tooth. Allow the material to set and then remove the band to investigate the impression. ${ }^{7}$ Fig. 2 


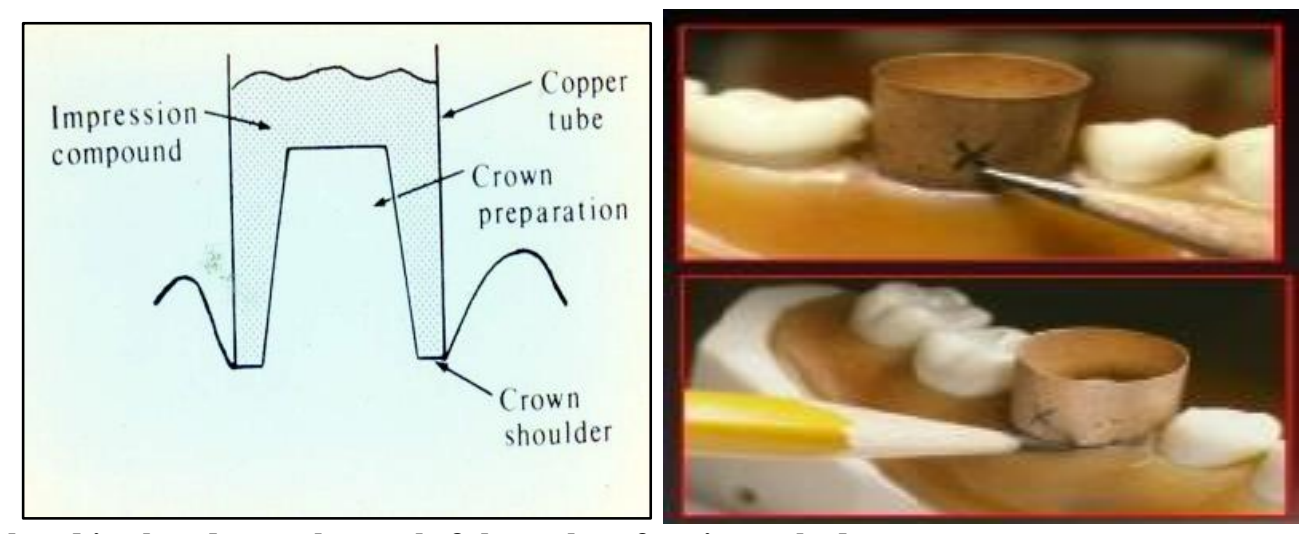

Fig. 2: Copper band is placed onto the tooth \& buccal surface is marked

\section{Monophase/ Single Viscosity Technique}

It is a simple technique \& easy to use, with excellent handling properties. But has disadvantages including more polymerization shrinkage than heavy body.

Procedure: Medium-viscosity impression materials of polyether or addition silicone with resin custom tray of 2 $4 \mathrm{~mm}$ spacing are often incorporated for impression making using the monophase technique. The medium-viscosity material is strained through the syringe reducing its viscosity 'shear thinning effect' whereas the viscosity of same material in the tray remains unaffected. Success depends on the pseudo plastic properties of these two materials. ${ }^{8}$

\section{Dual Viscosity Technique}

Impressions are made with low-viscosity and highviscosity impression materials of addition silicone with resin custom tray having 2 to $4 \mathrm{~mm}$ spacing for impression material In this technique a high-viscosity material is mixed and placed in impression tray while low-viscosity material is injected with a syringe onto the tooth surface. After injecting the low-viscosity material, tray loaded with high-viscosity material should be placed in patient's mouth. While the operator injects the light body material around the preparation margin, the assistant must mix the heavy body material and load into the tray and will give to the operator. This way the work becomes easy and the quality of impression is also improved. ${ }^{8}$

\section{E. Dual Arch Impression Technique}

Synonyms: Dual quad tray, triple arch, accu -bite, closed mouth impression, close bite double arch method.

The dual-arch technique captures the prepared teeth, the opposing arch, and the occlusal articulation in maximum intercuspation simultaneously. This technique should be used with a maximum of two prepared teeth and only with patients that have existing anterior guidance For this technique, the patient must be able to close completely in MIP with the impression tray in the mouth. Rigid metal trays are ideal for success. It requires the articulator to provide for a positive stop or there should be sufficient teeth to maintain the vertical dimension. There should be sufficient space distal to the terminal tooth in the arch to allow tray approximation.
Advantages of this technique involves less impression material, comfortable for the patient as less gagging occurs, and disadvantages like the tray used is not rigid and can only be used for one casting per quadrant. ${ }^{9}$ Its various technique includes:

a) Dual-arch multiple mix technique: Evaluation of the fit of the tray in the patient's mouth is done after checking bilateral closure. Rehearse till the patient is able to intimate the task in a similar manner. The final impression is made by mixing the low viscosity material and loaded in the syringe. The high viscosity material is loaded in the tray. After the low viscosity material is syringed the tray containing high viscosity material is positioned on the arch.

Procedure: Instruct the patient to close the mouth and observe for the interdigitation on the opposite arch and wait for the material to set. Instruct the patient to open the mouth remove the tray by applying equal pressure bilaterally and evaluate the impression. ${ }^{9}$

b) Dual-arch monophase technique: In single viscosity/monophase, impressions are often taken with medium-viscosity impression materials. Single mix with part of the material loaded in tray and another portion loaded in the syringe. When medium-viscosity material is forced through the syringe the viscosity is reduced whereas viscosity of same material in the tray is unaffected. After the low viscosity material is syringed the tray is positioned on the arch.

Procedure: Instruct the patient to close the mouth and observe for the interdigitation on the opposite arch and wait for the material to set. Direct the patient to open the mouth and remove the tray by applying equal pressure bilaterally. Check for the impression. ${ }^{9}$

c) Laminar impression technique: The laminar impression technique is a precise, rapid, and predictable alternative to traditional methods of impression-making in fixed prosthodontics.

Procedure: A preliminary impression is made using plastic "triple-arch" type trays and high-stiffness vinyl polysiloxane jaw relation registration material This registration can be used for provisional fabrication after tooth preparation, tissue management, and retraction. Then two holes are drilled through the facial wall of the tray into the preparation region. 
The tray is re-positioned in the mouth and light-bodied vinyl polysiloxane impression material is injected into the holes using an "auto-mixing" gun system. ${ }^{10}$

d) Hydraulic pressure technique: This technique is recommended to capture crown preparation margins without the need for gingival retraction.

Procedure: The preoperative impression of the unprepared tooth is made and a wash impression is recorded with a low or medium viscosity impression material around the prepared tooth and the operator reinsert the preoperative impression while the patient is asked to close into maximum intercuspation. The wash material if forced into the sulcus and around the preparation by the generation of hydraulic pressure forces. On the buccal or lingual aspect of the impression material and the tray vent holes are made to allow the escape of excess wash material. ${ }^{11}$

\section{F. Segmental Impression Technique}

The segmental impression technique proposes a procedure that unsurprisingly permits making a successful impression with multiple prepared teeth. The arch to be impressed is broken down into easily managed segments with this technique. The technique can be used with any impression material but is optimally used with auto-mix PVS material. $^{3}$

Procedure: One of the segmental trays and a syringe is loaded with light body. Now the impression material is injected around the preparations and the tray with the material is seated into the place and excess extruded material is removed from and around the tray. Allow the impression material to set. Do not remove the tray. Until each segmental impression is in place this procedure is repeated with each of the segments. After that an over impression is made with a compatible impression material in a stock tray Individual custom trays for each segment with $1 \mathrm{~mm}$ of wax spacer are used on the diagnostic cast. The tray should extend $3 \mathrm{~mm}$ past gingival margin of the prepared teeth because there are no occlusal stops and the gingival tissue must to prevent overseating of the trays and the PMMA custom tray made $24 \mathrm{hrs}$ in advance. Both segmented custom trays are selected with $1 \mathrm{~mm}$ spacer to take multiple prepared teeth and stock trays are used to take impression. ${ }^{12}$

\section{G. Wet Technique}

This impression technique requires a special conditioning unit which consists of 3 thermostatically controlled water baths.

Procedure: Select the correct size of water cooled impression trays and place small modeling compound or prefabricated stops in the tray to prevent over seating. Fill the impression tray with heavy bodied material from the storage bath and place it in the tempering bath. Now Load the syringe material in the syringe and carefully remove the retraction cord and flood the tooth with water and inject the syringe material on the tooth and cover the entire tooth. Remove the impression tray from the bath and wipe it with the gauze and place it in the mouth. After seating, cold water is circulated through the tray until the impression material is set. Remove the impression with a rapid motion, wash with cold water, evaluate for accuracy. ${ }^{13}$

\section{H. Hydrocolloid laminate technique}

It has certain merits like more accurate surface, more compatibility with gypsum, elimination of water-cooled impression trays, economical than newer elastomeric impression materials but at the same time it is stiff and therefore difficult to remove without rocking, has low tear resistance.

Procedure: This technique has two components, one is syringe agar which is injected around preparation. Second is the chilled alginate mix contained in the tray, promptly seated on the top of agar. ${ }^{14}$ Fig. 3

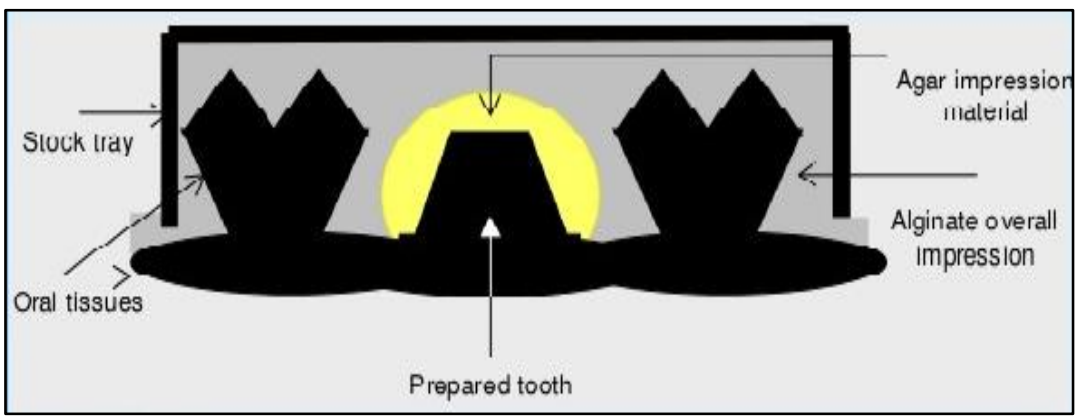

Fig. 3: Hydrocolloid laminate technique

\section{Impression using Polycarbonate Crowns}

The advantages of this technique include less potential for soft tissue trauma since gingival displacement is achieved with preformed shell, also lesser polymerization shrinkage due to small bulk of impression material within the preformed crowns. Indicated for complete arch impression of each prepared tooth involving gingival terminations.

Procedure: In this technique a final over impression is obtained using a stock tray and prefabricated temporary crown shells are used for each tooth preparation. Alterations are done in the gingival margin and proximal contacts and an adhesive is coated in the internal and external surfaces of provisional crowns. Then mix regular body \& crown shell is filled with it. While filling avoid entrapment of air and seat the crown shell until it covers the finish line. Wait for the material to set and finally make a pick up impression using regular body in a complete arch stock tray. ${ }^{15}$ 


\section{J. Functional Check-Bite Technique}

It is a combined record used for making inlays, crowns, and fixed partial dentures. Because of its simultaneous recording of prepared teeth and their precise relationship to the opposing teeth, the cast restorations made from such a record need less adjusting in the mouth than castings made from separate impressions and interocclusal records.

Procedure: The "check-bite-impression" obtained is an accurate static record of the teeth and their occlusal relationship. After the gingival packing has been removed and it is certain that the abutments are dry, the impression material from the syringe is injected over and around the preparations, the filled tray positioned in the mouth, and the patient is instructed to bite. The impression material is allowed to cure until prodding with an instrument shows that it is set, at that point of time the patient is told to "open."16

\section{K. Sectional Impressions and 'Every other Tooth' Technique in FPD}

The health of the tissues must be maintained while displacing gingiva with retraction cords. In case of multiple anterior tooth preparations with close root proximity, placement of the retraction cord simultaneously around all teeth results in strangulation of the interdental papillae. This can impair gingival health as well as cause black inter-dental triangles. To avoid this, 'every other tooth' technique of gingival displacement in combination with sectional impressions can be used. It will provide a better master cast in multiple tooth preparations. ${ }^{17}$

\section{Matrix Impression System}

A series of three impression procedures are required in this newer system. It uses three types and/or viscosities of impression materials. It strives to overcome the imperfections of the older systems and at the same time incorporate their best features. The matrix system effectively controls the four forces (relapsing, retraction, displacement, and collapsing) that impact on the gingiva during the phase of making the impression when attempting to register the sub gingival margins. The design of the matrix also gently forces the high viscosity impression material along the preparations and into the sulcus where it cleanses the sulcus of unwanted debris and fills the sulcus. The high viscosity material gently extends the sulcus and does not permit it to collapse as the medium viscosity material in the stock tray is seated for the pick-up impression. The matrix facilitates the formation of the optimum flange. Tearing is virtually eliminated because of the improved configuration of the sulcular flange and by the elimination of voids or contaminants in the sulcus.

Procedure: Over the tooth preparations a matrix of occlusal registration elastomeric material is made. Depending on the distribution and complexity of the preparations the matrix may be made in one piece or in two or more sections. The matrix is trimmed to prescribed dimensions and, after retraction cord is removed, a definitive impression is made in the matrix of the preparation with a high viscosity elastomeric impression material. While the matrix impression is in patients mouth, a stock tray filled with a medium viscosity elastomeric impression material is seated over the matrix and remaining teeth for creating an impression of the entire arch. $^{18}$

\section{Recent Technique \\ Digital impressions}

Digital impressions have evolved tremendously with time. The starting point for all systems is the capture of an accurate digital impression. Scanners are vehicles for obtaining dental images in dental computer-aided design/computer-aided manufacturing (CAD/CAM) systems for constructing fixed dental prostheses. CAD/CAM systems are available that either digitally scan and create fixed restorations chairside or that capture chairside digital impressions that are then sent to a laboratory. A virtual threedimensional (3D) model is created based on 3 sets of data (stone cast, impression body, and intraoral) acquired from the scanning process.

The ability to capture impressions digitally has been beneficial in the cases of a patient who is a gagger or cannot tolerate impression material in his or her mouth for several minutes, or if mandibular or maxillary tori or other undercuts are present that would make removal of a traditional impression cumbersome without causing the patient discomfort and/or tearing the margins on the impression. ${ }^{19}$

a) Chairside production: All components of the CAD/CAM system are located in the dental surgery. Fabrication of dental restorations can thus take place at chairside without a laboratory procedure. The digitalization instrument is an intra-oral camera, which replaces a conventional impression in most clinical situations. This saves time and offers the patient indirectly fabricated resto- rations at one appointment. At present, only the Cerec ${ }^{\circledR}$ System (Sirona) offers this possibility. ${ }^{20}$

b) Laboratory production: This variant of production is the equivalent to the traditional working sequence between the dentist and the laboratory. The dentist sends the impression to the laboratory where a master cast is fabricated first. The remaining $\mathrm{CAD} / \mathrm{CAM}$ production steps are carried out completely in the laboratory. With the assistance of a scanner, three-dimensional data are produced on the basis of the master die. These data are processed by means of dental design software. After the CAD-process the data will be sent to a special milling device that produces the real geometry in the dental laboratory. Finally, the exact fit of the frame- work can be evaluated and, if necessary, corrected on the basis of the master cast. The ceramist carries out the veneering of the frameworks in a powder layering or overpressing technique. ${ }^{21-24}$

c) Centralised production: In this variation, it is possible for 'satellite scanners' in the dental laboratory to be connected with a production centre via the Internet. Data sets produced in the dental laboratory are sent to the production centre for the restorations to be produced with a CAD/CAM device. Finally, the production centre sends the prosthesis to the responsible laboratory. Thus, production steps 1 and 2 take place in the dental laboratory, while the third step takes place in the centre..$^{22,25}$ 


\section{Discussion}

This article discuses significance of spacers used in fixed partial dentures \& various impression technique for the fabrication of successful FPD. The favorable as well as the unfavorable points of each technique were analyzed and various approaches were described in order to eliminate most of the unfavorable points during recording of void-free impressions of tooth preparations for fixed prosthodontics. Optimal space can be achieved by advocating spacers that will provide adequate space for the impression materials to achieve a good impression. The impressions made from 2 to $4 \mathrm{~mm}$ spaced trays produced more accurate impressions. It is therefore advisable not to exceed the tray space of $2 \mathrm{~mm}$ for any elastomeric impression materials tested.

The dentist must also select impression materials that are appropriate for each case and will provide the greatest chances of success. The impression procedure must be performed flawlessly and the final impression must be inspected for voids and defects. If any defect is suspected, the problem should be rectified and the impression should be remade.

\section{Conclusion}

An accurately fitting restoration requires a good quality impression that depends upon skill of the operator and accurate impression techniques used. Numerous factors should be considered while making of a definitive impression, all of which must be considered and studied properly to obtain an acceptable result accurately. Distortion of impression maybe caused by the kind of material selected, type of tray used, impression techniques, storage conditions, excessive seating pressure, too slow removal from the mouth etc. Good knowledge of material science, proper manipulation technique allows all the impression materials to create a negative form of teeth and oral tissues, into which gypsum and other die materials can be processed to create working analogues i.e. casts and study models. Currently poly vinyl siloxane and polyether are the most accurate. Thus the role of the operator is to select the appropriate technique for a particular clinical condition, considering the availability of trays, different impression materials, the time required, the salivary flow-its interference and management, the span of the restoration whether single unit crown or short span bridges or a long span bridgework, the critical areas to be recorded and finally the economy of the patient, all in harmonious relationship of the hard and soft tissue structures of the tooth.

\section{References}

1. Devan MM. Basic principles in impression making. J Prosthet Dent. 1952;2:26-35.

2. Livaditis GJ. Comparision of the new matrix system with traditional traditional fixed prosthodontic procedures. $J$ Prosthet Dent. 1998;79(2):200-207.

3. Donovan TE, Chee WWL. A review of contemporary impression materials and techniques. Dent Clin North Am. 2004;48(2):445-470.

4. Kumar V, Aeran H. Evaluation of Effect of Tray Space on the Accuracy of Condensation Silicone, Addition Silicone and
Polyether Impression Materials: An In Vitro Study. J Ind

Prosthodont Soc. 2012; 12(3):154-160.

5. Rosentiel SF, Land MF, Fujimoto J. contemporary fixed prosthodontics, $4^{\text {th }}$ ed

6. Cho GC, Chee WW. Distortion of disposable plastic stock trays when used with putty vinyl polysiloxane impression materials. J Prosthet Dent. 2004;92(4):354-358.

7. Kimmelman BB, Lerman. H. Impressions of single preparations using a copper band-shell. J Prosthet Dent. 1971;26(2):154-158.

8. Caputi S, Varvara G. Dimensional accuracy of resultant casts made by a monophase, one-step and two-step, and a novel twostep putty/light body impression technique. J Prosthet Dent. 2008;99(4):274-281.

9. Breeding LC, Dixon DL. Accuracy of casts generated from dual arch impressions. J Prosthet Dent. 2000;84(4):403-407.

10. Kaplowitz GJ. Trouble-shooting dual arch impressions. J Am Dent Assoc. 1996;127(2):234-240.

11. Gardener K, Loft GH. An intraoral coping technique for making impressions of multiple preparations. J Prosthet Dent. 1981;45(5):570-571.

12. Livaditis GJ. The matrix impression system for fixed prosthodontics. J Prosthet Dent. 1998;79(2):208-216

13. Dimashkieh MR1, Morgano SM. A procedure for making fxed prosthodontic impressions with the use of preformed crown shells. J Prosthet Dent. 1995;73(1):95-96.

14. Getz E.H. Functional ,checkbite-impressions” for fixed prosthodontics. J Prosthet Dent. 1971;26(2):146-153.

15. Hummert TW, Kaiser DA. Block-out technique for impressions of teeth with increased open gingival embrasures. J Prosthet Dent. 1999;82(1):100-102.

16. Livaditis GJ. Comparision of the new matrix system with traditional fixed prosthodontic impression procedures. $J$ Prosthet Dent. 1998;79(2):200-207.

17. Sudhapalli S. Sectional Impressions and 'Every Other Tooth' Technique in FPD. J Clin Diagn Res. 2017;11(1):ZD18-20.

18. Reed HV. Reversible agar agar hydrocolloid. Quintessence Int. 1990;21(3):225-229.

19. Lee W-S, Kim W-C, Kim H-Y, Kim W-T, Kim J-H. Evaluation of different approaches for using a laser scanner in digitization of dental impressions. J Adv Prosthodont. 2014;6(1):

20. F. Beuer, J. Schweiger and D. Edelhoff. Digital dentistry: an overview of recent developments for CAD/CAM generated restorations. Br Dent J. 2008;204(9):505-511.

21. Luthy H, Filser F, Loeffel O, Schumacher M. Strength and reliability of four unit all-ceramic posterior bridges. Dent Mater. 2005;21:930-937.

22. May K B, Russell M M, Razzoog M E, Lang B R. Precision of fit: the Procera AllCeram crown. J Prosthet Dent. 1998;80:394-404.

23. Raigrodski A J. All-ceramic full-coverage restorations: concepts and guidelines for material selection. Pract Proced Aesthet Dent. 2005;17:249-256.

24. Raigrodski A J, Chiche G J. The safety and efficacy of anterior ceramic fixed partial dentures: a review of the literature. $J$ Prosthet Dent. 2001;86:520-525.

25. Reich S, Wichmann M, Nkenke E, Proeschel P. Clinical fit of all-ceramic three-unit fixed partial dentures, generated with three different CAD/CAM systems. Eur J Oral Sci. 2005;113:174-179.

How to cite this article: Kumar V, Seth J, Sagar M, Aeran V. Anatomization of various impression techniques in fixed partial prosthodontics. Int $\mathbf{J}$ Oral Health Dent. 2018;4(4):208-213. 